\title{
Width and Partial Widths of Unstable Particles
}

\author{
Pietro A. Grassi, ${ }^{1}$ Bernd A. Kniehl, ${ }^{2}$ and Alberto Sirlin ${ }^{1}$ \\ ${ }^{1}$ Department of Physics, New York University, 4 Washington Place, New York, New York 10003 \\ ${ }^{2}$ II. Institut für Theoretische Physik, Universität Hamburg, Luruper Chaussee 149, 22761 Hamburg, Germany
}

(Received 29 August 2000)

\begin{abstract}
In the gauge theory context, a definition of branching ratios and partial widths of unstable particles is proposed that satisfies the basic principles of additivity and gauge independence. A simpler definition, similar to the conventional one, is examined in the $Z^{0}$-boson case. In order to establish contact with experiment, we show that it leads to a peak cross section that justifies the expression used by the LEP Electroweak Working Group through next-to-next-to-leading order, provided that the pole rather than the on-shell mass and width of the $Z^{0}$ boson is employed.
\end{abstract}

DOI: 10.1103/PhysRevLett.86.389

The mass, width, and partial widths of unstable particles rank among the basic concepts in particle physics. In fact, most fundamental particles of nature are unstable, and their masses, widths, and partial widths are some of their crucial defining properties. Yet, the precise and consistent definitions of these concepts have been notoriously difficult and elusive over a period spanning several decades. The reason is that unstable particles are not asymptotic states and, consequently, they lie somewhat outside the traditional formulation of quantum field theory.

The conventional definitions of mass and width are

$$
\begin{gathered}
M^{2}=M_{0}^{2}+\operatorname{Re} A\left(M^{2}\right), \\
M \Gamma=-\frac{\operatorname{Im} A\left(M^{2}\right)}{1-\operatorname{Re} A^{\prime}\left(M^{2}\right)},
\end{gathered}
$$

where $M_{0}$ is the bare mass and $A(s)$ is the self-energy in the case of scalar bosons and the transverse self-energy in the case of vector bosons. The partial widths are then defined by decomposing the numerator of Eq. (2) into a sum of contributions involving distinct sets of final-state physical particles. Most calculations of partial and total widths are based on Eqs. (1) and (2). We will refer to $M$ as the on-shell mass and to Eqs. (1) and (2) as the conventional on-shell formulation.

The emergence of gauge theories has brought into the discussion a new and powerful element, namely, the requirement of gauge independence of physical observables. It was shown in Ref. [1] that, in a gauge theory, Eqs. (1) and (2) become gauge dependent in $O\left(g^{4}\right)$ and $O\left(g^{6}\right)$, respectively, where $g$ is a generic gauge coupling. As the leading contributions to $M^{2}$ and $\Gamma$ are of order $O\left(g^{0}\right)$ and $O\left(g^{2}\right)$, respectively, we see that in both cases the problem arises in the next-to-next-to-leading order (NNLO). In the same paper, it was proposed that the way of solving this predicament is to base the definitions of mass and width on the complex-valued position of the propagator's pole,

$$
\bar{s}=M_{0}^{2}+A(\bar{s}),
$$

an idea that goes back to well-known tenets of $S$-matrix theory [2]. A frequently employed parametrization is $\bar{s}=m_{2}^{2}-i m_{2} \Gamma_{2}$, where we use the notation of Ref. [1].
PACS numbers: 11.10.Gh, 11.15.-q

Identifying $m_{2}$ and $\Gamma_{2}$ with the gauge-independent definitions of mass and width of the unstable particle, it follows from Eq. (3) that

$$
m_{2} \Gamma_{2}=-\operatorname{Im} A(\bar{s}) .
$$

Over the past several years, a number of authors have advocated the use of $\bar{s}$ as the basis for the definition of mass and width [3]; the conclusions of Ref. [1] have been confirmed by later studies [4,5] and proven to all orders [6]. It has been shown that, in the case of a heavy Higgs boson, the gauge dependences of $M$ and $\Gamma$ are numerically large [5]. It has also been emphasized that the on-shell definition of width [Eq. (2)] leads to severe problems if $A(s)$ is not analytic in the neighborhood of $M^{2}$. This occurs, for instance, when the mass of the decaying particle lies very close to a physical threshold $[7,8]$ or, in the resonance region, when the unstable particle is coupled to massless quanta, as in the cases of the $W$ boson and the unstable quarks [9]. Significant progress has also been achieved in the treatment of unstable particles in the framework of the pinch technique [10].

An important issue that arises at this stage is the following: if Eq. (4) provides a consistent definition of width, what is the definition of partial widths? It must clearly satisfy two important properties: additivity, i.e., the sum of the partial widths must equal the total width [Eq. (4)], and gauge independence.

We consider the process $i \rightarrow Z^{0} \rightarrow f$, where $i$ and $f$ are initial and final states involving particles which are either stable or have negligible widths. The transverse part of the propagator is given by

$$
\mathcal{D}_{\mu \nu}=-i \frac{Q_{\mu \nu}}{s-\bar{s}-[A(s)-A(\bar{s})]},
$$

where $Q_{\mu \nu}=g_{\mu \nu}-p_{\mu} p_{\nu} / s, p_{\mu}$ is the four-momentum of the $Z^{0}$ boson, and $s=p^{2}$. The vertex amplitude is of the form

$$
V_{f}^{\mu}(s) \equiv\left\langle f\left|J_{Z}^{\mu}\right| 0\right\rangle=\sum_{a} v_{f}^{(a)}(s, \ldots) M_{f}^{(a) \mu},
$$

where $M_{f}^{(a) \mu}$ denote various independent vector and axialvector matrix elements involving the spinors, polarization 
four-vectors, and four-momenta of the final-state particles, while $v_{f}^{(a)}(s, \ldots)$ are scalar functions. The dots indicate their additional dependence on the momenta of the finalstate particles. In this paper, we use the convention of including the coupling $-i g / c$, where $c$ is an abbreviation for $\cos \theta_{w}$, in the definition of $v_{f}^{(a)}(s, \ldots)$, so that, in leading order, $v_{f}^{(a)}(s, \ldots)=O(g)$. Expanding Eq. (6) and the denominator of Eq. (5) about $s=\bar{s}$, it is well known [3] that the overall amplitude can be written in the form

$$
\mathcal{A}_{f i}(s)=-i \frac{Q_{\mu \nu} V_{f}^{\mu}(\bar{s}) V_{i}^{\nu}(\bar{s})}{(s-\bar{s})\left[1-A^{\prime}(\bar{s})\right]}+N,
$$

where $N$ represents nonresonant contributions. As the pole residues $v_{f}^{(a)}(\bar{s}, \ldots) v_{i}^{(b)}(\bar{s}, \ldots) /\left[1-A^{\prime}(\bar{s})\right]$ are gauge independent for any choice of the states $i, f$ and the amplitudes $a, b$, a gauge-independent definition of partial width is given by

$$
m_{2} \hat{\Gamma}_{f}=-\frac{1}{6} \sum_{\text {spins }} \int d \Phi_{f} \frac{Q_{\mu \nu} V_{f}^{\mu *}(\bar{s}) V_{f}^{\nu}(\bar{s})}{\left|1-A^{\prime}(\bar{s})\right|} .
$$

The integration is over the phase space of the final-state particles with $\left(\sum_{n} p_{n}\right)^{2}=m_{2}^{2}$, a factor of $1 / 3$ arises from the average over the initial-state polarization, and a factor of $1 / 2$ arises from the familiar relation between $m_{2} \hat{\Gamma}_{f}$ and the integrated amplitude square.

A limitation of Eq. (8) is that there is no guarantee that it satisfies the additivity property. In fact, it is expected that $\sum_{f} \hat{\Gamma}_{f} \neq \Gamma_{2}$ when one includes NNLO contributions. In order to remedy this situation, we propose to define the branching ratios by

$$
B_{f}=\frac{\hat{\Gamma}_{f}}{\sum_{f} \hat{\Gamma}_{f}},
$$

and the partial widths by

$$
\Gamma_{f}=B_{f} \Gamma_{2} .
$$

The gauge independence of Eq. (8) implies that of Eq. (9), while Eqs. (9) and (10) guarantee the additivity property, $\sum_{f} \Gamma_{f}=\Gamma_{2}$. The rescaling in Eqs. (9) and (10) implies that $\hat{\Gamma}_{f}=\Gamma_{f}\left(X / m_{2} \Gamma_{2}\right)$, where

$$
X=m_{2} \sum_{f} \hat{\Gamma}_{f} .
$$

The resonant cross section at $s=m_{2}^{2}$ is proportional to $\hat{\Gamma}_{e} \hat{\Gamma}_{f} /\left(m_{2} \Gamma_{2}\right)^{2}=\left[\Gamma_{e} \Gamma_{f} /\left(m_{2} \Gamma_{2}\right)^{2}\right]\left(X / m_{2} \Gamma_{2}\right)^{2}$, where $\Gamma_{e}$ is the $Z^{0} \rightarrow e^{+} e^{-}$partial width. Hence, it is modified by a factor $\left(X / m_{2} \Gamma_{2}\right)^{2}$ when expressed in terms of the widths $\Gamma_{f}$ that satisfy the additivity property. We note that $X / m_{2}$ and $\Gamma_{2}$ represent two different definitions of total width, based on the pole residues and the pole position, respectively. The ratio $\left(X / m_{2} \Gamma_{2}\right)$ differs from unity by gauge-independent terms of $O\left(g^{4}\right)$, i.e., in NNLO. As a consequence, in the $Z^{0}$-boson case, this is expected to be a very small effect, of the same order of magnitude as nonresonant contributions that are frequently neglected.

Next, we examine the difference $X-m_{2} \Gamma_{2}$ in greater detail. It is convenient to split $I\left(m_{2}^{2}\right) \equiv \operatorname{Im} A\left(m_{2}^{2}\right)$ into the form

$$
\begin{gathered}
I\left(m_{2}^{2}\right)=F\left(m_{2}^{2}\right)+G\left(m_{2}^{2}\right), \\
F\left(m_{2}^{2}\right)=\sum_{f} I_{f}\left(m_{2}^{2}\right), \\
I_{f}\left(m_{2}^{2}\right)=\frac{1}{6} \sum_{\text {spins }} \int d \Phi_{f} Q_{\mu \nu} V_{f}^{\mu *}\left(m_{2}^{2}\right) V_{f}^{\nu}\left(m_{2}^{2}\right) .
\end{gathered}
$$

$-I_{f}\left(m_{2}^{2}\right) / m_{2}$ is the conventional expression for the partial width of the unstable particle into the physical state $f$, modulo the wave-function renormalization of the unstable particle, with the important difference that it is evaluated at the gauge-independent pole mass $m_{2}$ rather than the gauge-dependent on-shell mass $M$. $G\left(m_{2}^{2}\right) \equiv I\left(m_{2}^{2}\right)-F\left(m_{2}^{2}\right)$ involves contributions from unphysical intermediate states (Goldstone bosons, FaddeevPopov ghosts, and longitudinal modes of gauge bosons), which can contribute to $I\left(m_{2}^{2}\right)$ for sufficiently low values of the gauge parameters [1].

In the conventional on-shell formulation, it is assumed that $I\left(M^{2}\right)$ can be expressed as a sum of contributions involving solely physical intermediate states, namely, $I\left(M^{2}\right)=\sum_{f} I_{f}\left(M^{2}\right)$. The argument invokes the unitarity of the $S$ matrix and would, in fact, be valid if $I\left(M^{2}\right)$ were an $S$-matrix amplitude. However, as the unstable particle is not an asymptotic state, this is not the case, and the above decomposition into physical cut contributions must be viewed as an approximation. In fact, we will show in this section that $G\left(m_{2}^{2}\right) \neq 0$ in $O\left(g^{6}\right)$, i.e., in NNLO.

We study the difference $X-m_{2} \Gamma_{2}=X+\operatorname{Im} A(\bar{s})$, where $X$ is defined in Eqs. (8) and (11), by expanding $v_{f}^{(a)}(\bar{s}, \ldots), A^{\prime}(\bar{s})$, and $A(\bar{s})$ about $s=m_{2}^{2}$ through terms of $O\left(g^{6}\right)$. The leading term in the expansion of $\operatorname{Im} A(\bar{s})$ is $I\left(m_{2}^{2}\right)$, for which we employ the decomposition of Eq. (12). The $F\left(m_{2}^{2}\right)$ term cancels the leading contribution from $X$, and we find

$$
\begin{aligned}
X-m_{2} \Gamma_{2}= & \frac{\left(m_{2} \Gamma_{2}\right)^{2}}{2} I^{\prime \prime}-\frac{m_{2} \Gamma_{2}}{2}\left(I^{\prime}\right)^{2}+G\left(m_{2}^{2}\right) \\
& -\frac{m_{2} \Gamma_{2}}{3} \sum_{f \text { spins }} \int d \Phi_{f} Q_{\mu \nu} \\
& \times \operatorname{Im}\left[V_{f}^{\mu *}\left(m_{2}^{2}\right) V_{f}^{\nu \prime}\left(m_{2}^{2}\right)\right],
\end{aligned}
$$

where the primes indicate derivatives with respect to $s$, evaluated at $s=m_{2}^{2}$. Since $X$ and $m_{2} \Gamma_{2}$ are gauge independent, Eq. (15) determines the gauge-dependent part of $G\left(m_{2}^{2}\right)$ in $O\left(g^{6}\right)$. Since $\Gamma_{2}$ and $I$ are of $O\left(g^{2}\right), v_{f}^{(a)}$ is of $O(g)$, and $v_{f}^{(a) \prime}$ is of $O\left(g^{3}\right)$, it suffices to consider the gauge dependence of the one-loop electroweak contributions to $I(s)$ and $v_{f}^{(a)}(s, \ldots)$. Furthermore, in the 
consideration of the vertex contributions, we may restrict ourselves to the two-particle final states, since those involving more particles give gauge-dependent contributions of higher order.

In the $Z^{0}$-boson case, the gauge dependence of $I(s)$ and $v_{f}^{(a)}(s, \ldots)$ at the one-loop level can be obtained from Eqs. (7), (8), (17), and (24) of Ref. [11] in the approximation of neglecting the masses of the external fermions, which we henceforth adopt. Applying those results to Eq. (15) and noting that $G\left(m_{2}^{2}\right)$ vanishes for $\xi_{W}>1 / c^{2}$, where $\xi_{W}$ is the gauge parameter associated with the $W$ boson, we find

$$
\begin{aligned}
G\left(m_{2}^{2}\right)= & \frac{m_{2} \Gamma_{2}}{2}\left[\left(I^{\prime}\right)^{2}-\left(F^{\prime}\right)^{2}\right]-g^{2} c^{2} m_{2}^{2} \Gamma_{2}^{2}\left(\xi_{W}-1\right) \\
& \times \operatorname{Im} \eta_{W}\left(m_{2}^{2}\right)+O\left(g^{8}\right),
\end{aligned}
$$

where $\eta_{W}$ is a gauge-dependent amplitude given in Ref. [11]. Its imaginary part is nonvanishing in a subclass of gauges characterized by $M_{Z}>2 \sqrt{\xi_{W}} M_{W}$ or $\xi_{W}<1 /\left(4 c^{2}\right)[1,11]$. It is worth noting that one-loop $\gamma-Z$ mixing contributions, which have been taken into account, cancel in the derivation.

We now discuss an alternative and manifestly additive definition of branching ratios, namely,

$$
\tilde{B}_{f, 2}=\frac{I_{f}\left(m_{2}^{2}\right)}{F\left(m_{2}^{2}\right)} .
$$

The corresponding partial widths are

$$
\tilde{\Gamma}_{f, 2}=\tilde{B}_{f, 2} \Gamma_{2} .
$$

Equation (17) is the conventional definition employed in current calculations, except that the amplitudes are evaluated at the gauge-independent pole mass $m_{2}$ rather than at the on-shell mass $M$. Similarly, Eq. (18) also involves the gauge-independent width $\Gamma_{2}$ rather than $\Gamma$. Recalling Eq. (4), Eq. (8) can be expressed in the form

$$
m_{2} \tilde{\Gamma}_{f, 2}=-\frac{I_{f}\left(m_{2}^{2}\right)}{F\left(m_{2}^{2}\right)} \frac{I\left(m_{2}^{2}\right)}{1+\left[\operatorname{Im} A(\bar{s})-I\left(m_{2}^{2}\right)\right] /\left(m_{2} \Gamma_{2}\right)},
$$

since the second factor on the right-hand side equals $-m_{2} \Gamma_{2}$ [8]. We note that the denominator of this second factor differs from the conventional wave-function renormalization in NNLO. By construction, Eqs. (17) and (18) satisfy the additivity property, $\sum_{f} \tilde{\Gamma}_{f, 2}=\Gamma_{2}$. In order to establish contact with experiment, we now show that it leads to a peak cross section that is gauge independent through $O\left(g^{4}\right)$, i.e., through NNLO.

Using Eqs. (5) and (6), the amplitude at $s=m_{2}^{2}$ for the process $i \rightarrow Z^{0} \rightarrow f$ is found to be

$$
\mathcal{A}_{f i}\left(m_{2}^{2}\right)=-\frac{Q_{\mu \nu} V_{f}^{\mu}\left(m_{2}^{2}\right) V_{i}^{\nu}\left(m_{2}^{2}\right)}{m_{2} \Gamma_{2}-i\left[A(\bar{s})-A\left(m_{2}^{2}\right)\right]}+\tilde{N},
$$

where $\tilde{N}$ represents nonresonant contributions. Disregarding for the moment the contributions from $\tilde{N}$, we consider the square of the absolute value of the first term on the right-hand side of Eq. (20), integrate over the phase space of the final-state particles, sum over their spins, and average over those of the initial-state particles. Noting that $\operatorname{Re}\left[A(\bar{s})-A\left(m_{2}^{2}\right)\right]=m_{2} \Gamma_{2} I^{\prime}\left(m_{2}^{2}\right)=O\left(g^{4}\right)$ in leading order, and making use of Eqs. (12), (14), and (19), we find, for the resonant contribution through $O\left(g^{4}\right)$,

$$
\sigma_{R}^{0}=\frac{12 \pi \tilde{\Gamma}_{e, 2} \tilde{\Gamma}_{f, 2}}{m_{2}^{2} \Gamma_{2}^{2}}\left[1-\left(I^{\prime}\right)^{2}+\frac{2 G\left(m_{2}^{2}\right)}{m_{2} \Gamma_{2}}\right],
$$

where we have identified the initial state with an $e^{+} e^{-}$pair and $\tilde{\Gamma}_{e, 2}\left(\tilde{\Gamma}_{f, 2}\right)$ is the $Z^{0} \rightarrow e^{+} e^{-}\left(Z^{0} \rightarrow f\right)$ partial width, defined according to Eqs. (17) and (18). In Eq. (21), it is understood that $\sigma^{0}$ is the cross section devoid of initialstate radiation effects, which are usually taken into account by a suitable convolution with a radiator function [12].

At this stage, we recall that the nonresonant amplitude $\tilde{N}$ in Eq. (20) includes contributions from box and photon-mediated diagrams as well as nonresonant effects from $\gamma-Z$ mixing graphs. In leading order, their gaugedependent parts can be found from the results of Ref. [11]. Inserting Eq. (16) into Eq. (21), one finds that the gauge-dependent $\left(I^{\prime}\right)^{2}$ term is removed and that the $\operatorname{Im} \eta_{W}$ contribution in Eq. (16) cancels in leading order the gauge-dependent part of the interference of $\tilde{N}$ with the first term in Eq. (20). Thus, we find, at $s=m_{2}^{2}$,

$$
\sigma^{0}\left(m_{2}^{2}\right)=\frac{12 \pi \tilde{\Gamma}_{e, 2} \tilde{\Gamma}_{f, 2}}{m_{2}^{2} \Gamma_{2}^{2}}\left(1-\frac{\Gamma_{2}^{2}}{m_{2}^{2}}\right)+\sigma_{\mathrm{B}}^{0}\left(m_{2}^{2}\right),
$$

where we have used $F^{\prime}\left(m_{2}^{2}\right)=-\Gamma_{2} / m_{2}$ in leading order and the background part $\sigma_{B}^{0}$ is a gauge-independent contribution of $O\left(g^{4}\right)$. As $\sigma^{0}$ is a physical observable, the fact that Eq. (22) is devoid of gauge-dependent contributions implies that the partial widths $\tilde{\Gamma}_{e, 2}$ and $\tilde{\Gamma}_{f, 2}$, defined on the basis of Eqs. (17) and (18), are gauge independent through $O\left(g^{6}\right)$, i.e., through NNLO.

The current analyses of the electroweak data measure $m_{1}=\left(m_{2}^{2}+\Gamma_{2}^{2}\right)^{1 / 2}$ and $\Gamma_{1}=m_{1} \Gamma_{2} / m_{2}$ rather than $m_{2}$ and $\Gamma_{2}$ [1], and determine the peak cross section at $s=m_{1}^{2}$ rather than at $s=m_{2}^{2}$. If the branching ratios are defined by $\tilde{B}_{f, 1}=I_{f}\left(m_{1}^{2}\right) / F\left(m_{1}^{2}\right)$ and the partial widths by $\tilde{\Gamma}_{f, 1}=$ $\tilde{B}_{f, 1} \Gamma_{1}$, instead of Eqs. (17) and (18), we find that the cross section at $s=m_{1}^{2}$ is given by

$$
\sigma^{0}\left(m_{1}^{2}\right)=\frac{12 \pi \tilde{\Gamma}_{e, 1} \tilde{\Gamma}_{f, 1}}{m_{1}^{2} \Gamma_{1}^{2}}+\sigma_{\mathrm{B}}^{0}\left(m_{1}^{2}\right) .
$$

The theoretical expression employed by the LEP Electroweak Working Group (EWWG) [13] is of the same form as the first term in Eq. (23). Thus, Eq. (23) justifies this expression through terms of $O\left(g^{4}\right)$, i.e., through NNLO in the electroweak interactions, provided that the gaugeindependent definitions of mass and width are employed. We note that this means that contributions of $O\left(g^{6}\right)$ to the partial and total widths are incorporated into Eqs. (22) and (23). On the other hand, $\sigma_{\mathrm{B}}^{0}$ can be evaluated from tree-level and one-loop diagrams. 
We conclude with the following observations and summary of our results: (i) In the hadronic sector, the formulation of this paper is restricted to the parton level of quarks and gluons, i.e., the effects of confinement are not taken into account. Since $\Gamma_{\tau}$ is of order $10^{-3} \mathrm{eV}$ and the widths of the least massive $B$ and $D$ mesons are even smaller, we neglect the leptonic widths as well as those of the five lightest quarks. (ii) If the final-state particles have negligible widths, Eq. (8) provides a gaugeindependent definition of partial widths to all orders of perturbation theory. In Eqs. (9) and (10), we have shown how this definition can be modified in order to satisfy the additivity property. (iii) A rigorous analysis does not include unstable particles in the final states. They are rather treated as virtual particles, which decay into stable ones. Examples are $Z^{0} \rightarrow f_{1} \bar{f}_{2} W^{*} \rightarrow f_{1} \bar{f}_{2} f_{3} \bar{f}_{4}$, $H \rightarrow W^{+*} W^{-*} \rightarrow f_{5} \bar{f}_{6} f_{7} \bar{f}_{8}$, where $f_{i}$ denote stable fermions. In domains of phase space where the virtual $W^{*}$ bosons are in their resonant regions, a resummation analogous to Eq. (7) is in general required. In the $W$-boson case, processes of this kind are forbidden by kinematic considerations. (iv) In Eq. (15), we have analyzed, to leading order and in the $Z^{0}$-boson case, the difference $X-m_{2} \Gamma_{2}$ between two different gauge-independent definitions of total widths, based on the pole residues and the pole position, respectively. An interesting by-product is the evaluation of the amplitude $G\left(m_{2}^{2}\right)$ [Eq. (16)], which represents the contribution to $I\left(m_{2}^{2}\right)$ from nonphysical intermediate states. The result $G\left(m_{2}^{2}\right) \neq 0$ in $O\left(g^{6}\right)$ reflects the fact that the unstable particle is not an asymptotic state. (v) In Eqs. (17)-(23), we have examined, in the $Z^{0}$-boson case, an alternative and simpler definition of partial widths that is similar to the one employed in current calculations, except that it makes use of the pole rather than the on-shell mass and width. Subject to this modification, Eq. (23) provides a theoretical justification, through NNLO in the electroweak interactions, for the peak cross section employed by the EWWG [13]. In this regard, it is important to note that Eqs. (22) and (23) incorporate corrections of $O\left(g^{6}\right)$ to the width and partial widths.

A.S. thanks the members of the 2nd Institute for Theoretical Physics of Hamburg University and the Brookhaven National Laboratory for their warm hospitality during summer 2000, and the Alexander von Humboldt Foundation for its kind support. The research of P.A.G. and A.S. was supported in part by NSF Grants No. PHY-9722083 and No. PHY-0070787. The research of B. A. K. was supported in part by DFG Grant No. KN 365/1-1, by BMBF Grant No. 05 HT9GUA 3, and by the European Commission through TMR Network No. ERBFMRX-CT98-0194.

[1] A. Sirlin, Phys. Rev. Lett. 67, 2127 (1991); Phys. Lett. B 267, 240 (1991).

[2] R.E. Peierls, in The Proceedings of the 1954 Glasgow Conference on Nuclear and Meson Physics, edited by E.H. Bellamy and R.G. Moorhouse (Pergamon, New York, 1955), p. 296; M. Lévy, Nuovo Cimento XIII, 115 (1959); R. J. Eden, P. V. Landshoff, D. I. Olive, and J.C. Polkinghorne, The Analytic S-Matrix (Cambridge University Press, Cambridge, England, 1966), p. 247.

[3] M. Consoli and A. Sirlin, CERN Yellow Report No. 86-02, 1986, Vol. 1, p. 63; S. Willenbrock and G. Valencia, Phys. Lett. B 259, 373 (1991); R. G. Stuart, Phys. Lett. B 262, 113 (1991); 272, 353 (1991); Phys. Rev. Lett. 70, 3193 (1993); Nucl. Phys. B498, 28 (1997); H. Veltman, Z. Phys. C 62, 35 (1994); A. R. Bohm and N. L. Harshman, Nucl. Phys. B581, 581 (2000).

[4] M. Passera and A. Sirlin, Phys. Rev. Lett. 77, 4146 (1996).

[5] B. A. Kniehl and A. Sirlin, Phys. Rev. Lett. 81, 1373 (1998); Phys. Lett. B 440, 136 (1998).

[6] P. Gambino and P. A. Grassi, Phys. Rev. D 62, 076002 (2000).

[7] B. A. Kniehl, Nucl. Phys. B357, 439 (1991); T. Bhattacharya and S. Willenbrock, Phys. Rev. D 47, 4022 (1993).

[8] B. A. Kniehl, C. P. Palisoc, and A. Sirlin, Nucl. Phys. B591, 296 (2000).

[9] M. Passera and A. Sirlin, Phys. Rev. D 58, 113010 (1998).

[10] J. Papavassiliou and A. Pilaftsis, Phys. Rev. Lett. 75, 3060 (1995); Phys. Rev. D 53, 2128 (1996); 54, 5315 (1996); N. J. Watson, Nucl. Phys. B494, 388 (1997); J. Papavassiliou, E. de Rafael, and N. J. Watson, Nucl. Phys. B503, 79 (1997); J. Papavassiliou, in JHEP Proceedings of the Corfu Summer Institute on Elementary Particle Physics, 1998, edited by I. Antoniadis, G. Koutsoumbas, N. Tracas, and G. Zoupanos, Reports No. PRHEPcorfu98/076 and No. hep-ph/9905328 (unpublished).

[11] G. Degrassi and A. Sirlin, Phys. Rev. D 46, 3104 (1992).

[12] Particle Data Group, D. E. Groom et al., Eur. Phys. J. C 15, 256 (paragraph 1) (2000).

[13] The LEP Collaborations ALEPH, DELPHI, L3, OPAL, the LEP Electroweak Working Group, and the SLD Heavy Flavour and Electroweak Groups, D. Abbaneo et al., Report No. CERN-EP-2000-016, 2000; (URL: http://lepewwg.web.cern.ch/LEPEWWG/). 